科 学 通 报

\title{
取代芳烃类化合物定量结构与 活性相关研究*
}

\author{
何艺兵 刘征涛 赵元慧 王连生 $* *$
}

(南京大学环境科学与工程系, 南京 210008)

\section{关键词定量结构与活性相关、最高占有轨道能、辛醇/水分配系数}

定量结构活性相关 (QSARs) 法起始于药物和杀虫剂研究 ${ }^{[1]}$, 它是通过研究有机物生物活 性与结构理化参数之间的相关性来预测新有机物的生物活性, 指导新药及杀虫剂的合成, 从 而减少了不必要的摸索, 节省了大量的费用和时间.

随着工业的发展, 每年数以万计的有机物排人环境中, 要全面评价众多的有机污染物在环 境中的行为是很难办到的 ${ }^{[2]}$. 到了 70 年代, 环境科学工作者将定量结构活性相关法引人了环 境污染物毒性研究, 其中具有代表性的方法有: 辛醇/水分配系数法、线性溶剂化能相关法和 分子连接性指数法 ${ }^{[1]}$, 然而这些方法都存在毒性机理不明确, 参数选择带有一定的盲目性等问 题. 近年来, 应用一级反应动力学模型研究水生生物中毒机理表明 ${ }^{[3,4]}$, 有机物在水和生物体之 间的分配及进人生物体的有机物分子与生物体分子间的电子相互作用导致了水生生物中毒. 本文在此基础之上结合量子化学知识, 以大型蛋为研究对象, 试从理论上初步探讨生物中毒、 机理.

\section{1 材 料 与 方 法}

\section{1 仪器}

恒温水浴缸 (自制), WMZK-01 型温度控制仪 (上海医用仪表厂)

\section{2 大型蛋半数致死浓度及效应浓度测定}

本文选用水生生物大型蛋 (Daphnia magna straus) 为受试物, 测定 $48 \mathrm{~h}$ 的半致 (LC50) 和 效应浓度 (EC50), 测定方法参见文献 $[5,6]$.

\section{3 理化参数}

试验化合物的辛醇 / 水分配系数 $\left(K_{\mathrm{ow}}\right)$ 取自文献 [7] 及碎片估算法 ${ }^{[2]}$, 最高占有轨道能 $\left(E_{\text {номо }}\right)$ 由 $\mathrm{HMO}$ 法计算得到.

\section{2 理 论 部 分}

研究证明, 水生物对有机物的吸收与释放符合一级反应动力学模型 ${ }^{[3]}$ :

1993-11-16 收稿, 1994-03-15 收修收稿.

*国家自然科学基金资助项目.

***联系人. 


$$
\mathrm{d} C_{\mathrm{s}} / \mathrm{d} t=k_{1} C_{\mathrm{w}}-k_{2} C_{\mathrm{s}},
$$

$k_{1}, k_{2}$ 是水生物对有机物的吸收和释放常数, $C_{\mathrm{S}}$ 和 $C_{\mathrm{w}}$ 分别是试验有机物在生物体内和水中 浓度. 对 (1) 式积分得 $t$ 时生物体内浓度:

$$
C_{\mathrm{S}}=C_{\mathrm{w}}\left(k_{1} / k_{2}\right)\left(1-e^{-k_{2} t}\right)+C_{\mathrm{S}}(\mathrm{o}) e^{-k_{2} t} .
$$

根据毒性试验条件, $t=0$ 时, $C_{\mathrm{S}}(\mathrm{o})=0, t=T$ 时, $50 \%$ 大型蛋体内有机物浓度达到临界致死浓 度, 此时水相中有机物浓度为 LC50, 则由 (2) 式得

$$
\mathrm{LC} 50=C_{\mathrm{S}} /\left(k_{1} / k_{2}\right)\left(1-e^{-k_{2} t}\right),
$$

(3) 式中 $k_{1} / k_{2}=\mathrm{BCF}, \operatorname{lgBCF}=a \lg K_{\mathrm{OW}}+b a, b$ 为常数 ${ }^{[8]}$. 对于有机物分子较小, 时间足够长的 情况下, $1-e^{-k_{2} T} \approx 1^{[4]}$, 毒性数据一般表示为 $\lg l / L C 50$, 则由 (3) 式得

$$
\lg 1 / \mathrm{LC} 50=a \lg K_{\mathrm{ow}}-\lg C_{\mathrm{s}}+C,
$$

式中 $c$ 为常数项, $C_{\mathrm{s}}=\mathrm{N} / V N_{\mathrm{O}} . N$ 为进人生物体内的有机物分子数, $N_{\mathrm{O}}$ 为阿佛加德罗常数, $V$ 为生物体的体积, 根据统计热力学原理,

$$
C_{\mathrm{S}}=e^{m} e^{n E_{\mathrm{o}}} / V N_{\mathrm{O}},
$$

\begin{tabular}{|c|c|c|c|c|c|c|c|c|}
\hline \multirow{2}{*}{ 化合物 } & \multirow{2}{*}{$\lg K_{\text {ow }}$} & \multirow{2}{*}{$\begin{array}{c}E_{\text {номо }} \\
(\beta)\end{array}$} & \multicolumn{3}{|c|}{$\lg 1 / \mathrm{LC} 50(\mathrm{~mol} / \mathrm{L})$} & \multicolumn{3}{|c|}{$\lg 1 / \mathrm{ECS} 0(\mathrm{~mol} / \mathrm{L})$} \\
\hline & & & 测定值 & 计算值 & 残差 & 测定值 & 计算值 & 残差 \\
\hline 1 苯 & 2.12 & 1.00 & 3.15 & 3.04 & 0.11 & 3.31 & 3.23 & 0.08 \\
\hline 2 氯苯 & 2.84 & 0.950 & 3.37 & 3.50 & -0.13 & 3.59 & 3.68 & -0.09 \\
\hline 31,2 二二唡苯 & 3.38 & 0.923 & 3.59 & 3.82 & -0.23 & 3.83 & 4.00 & -0.17 \\
\hline 4 1,3-二氯苯 & 3.38 & 0.927 & 3.64 & 3.82 & -0.18 & 3.84 & 3.99 & -0.15 \\
\hline 5 1,4 二氯苯 & 3.39 & 0.904 & 3.65 & 3.87 & -0.22 & 3.91 & 4.03 & -0.12 \\
\hline 6 1,2,3-三埭苯 & 4.24 & 0.923 & 4.30 & 4.25 & 0.05 & 4.54 & 4.42 & 0.12 \\
\hline 7 1,2,3,4 四象苯 & 5.03 & 0.880 & 4.66 & 4.73 & -0.07 & 4.84 & 4.89 & -0.05 \\
\hline 8 五氯苯 & 5.69 & 0.860 & 5.23 & 5.10 & 0.13 & 5.49 & 5.25 & 0.24 \\
\hline 9 六爭苯 & 6.41 & 0.860 & 5.31 & 5.46 & -0.15 & 5.51 & 5.61 & -0.10 \\
\hline 10 氛苯 & 2.61 & 0.923 & 3.47 & 3.44 & 0.03 & 3.67 & 3.60 & 0.07 \\
\hline 11 澳苯 & 2.79 & 0.946 & 3.61 & 3.48 & 0.13 & 3.81 & 3.66 & 0.15 \\
\hline 124 氯溴苯 & 3.72 & 0.903 & 3.78 & 4.03 & -0.25 & 4.04 & 4.20 & -0.16 \\
\hline 13 3-埭溴苯 & 3.83 & 0.925 & 4.15 & 4.04 & 0.11 & 4.39 & 4.21 & 0.18 \\
\hline 14 2-氯溴苯 & 3.83 & 0.921 & 4.16 & 4.05 & 0.11 & 4.37 & 4.22 & 0.15 \\
\hline 151,2 -二埧苯 & 4.07 & 0.920 & 4.03 & 4.17 & -0.14 & 4.28 & 4.34 & -0.06 \\
\hline $161,3-$ 二溴苯 & 3.81 & 0.924 & 4.11 & 4.04 & 0.07 & 4.35 & 4.21 & 0.14 \\
\hline 171,4 二澳苯 & 4.25 & 0.901 & 4.42 & 4.30 & 0.12 & 4.66 & 4.47 & 0.19 \\
\hline 18 苯胺 & 0.95 & 0.544 & 3.25 & 3.37 & -0.12 & 3.41 & 3.48 & -0.07 \\
\hline 19 2- 氯苯胺 & 1.83 & 0.542 & 3.80 & 3.81 & -0.01 & 3.97 & 3.92 & 0.05 \\
\hline 204 氯苯胺 & 1.90 & 0.523 & 3.90 & 3.89 & 0.01 & 4.01 & 3.99 & 0.02 \\
\hline 21 苯酚 & 1.46 & 0.792 & 3.06 & 3.13 & -0.07 & 3.26 & 3.28 & -0.02 \\
\hline 224 氯苯酚 & 2.39 & 0.756 & 3.69 & 3.66 & 0.03 & 3.93 & 3.81 & 0.12 \\
\hline 23 2-氯苯酚 & 2.18 & 0.772 & 3.54 & 3.53 & 0.01 & 3.75 & 3.68 & 0.07 \\
\hline
\end{tabular}

$m, n$ 为拉格兰吉因子, $E_{\mathrm{O}}$ 为分子基态能量, 前沿轨道理论认为前沿轨道中的电子在化学反应

表 1 毒性数据和参数 ${ }^{\mathrm{a})}$

a) 本表只列出部分数据. 
中起着极为重要的作用, 所以对于基态分子只取最高占有轨道能量 $\left(E_{\mathrm{HOMO}}\right)$ 则得

$$
C_{\mathrm{S}}=\left(K^{\text {епЕ номо }}\right) / V N_{\mathrm{O}},
$$

$K$ 为常数, 将(6) 式代人 (4) 式中整理得

$$
\lg 1 / \mathrm{LC} 50=a_{1} \lg K_{\text {оw }}-b_{1} E_{\text {номо }}+c_{1},
$$

$a_{1}, b_{1}, c_{1}$ 为常数. 同理可得

$$
\lg 1 / \mathrm{EC} 50=a_{2} \lg K_{\mathrm{Ow}}-b_{2} E_{\text {номо }}+c_{2},
$$

$a_{2}, b_{2}, c_{2}$ 为常数.

\section{3 结果与 讨 论}

对表 1 毒性数据和理化参数用方程 (7) 和 (8) 分别进行回归分析, 结果为

$$
\begin{gathered}
\lg 1 / \mathrm{LC} 50=0.498 \lg K_{\mathrm{Ow}}-2.00 E_{\text {номо }}+3.99, \\
n=35, \quad R^{2}=0.918 . \\
\lg 1 / \mathrm{EC} 50=0.494 \lg K_{\mathrm{OW}}-1.81 E_{\text {номо }}+4.00, \\
n=35, \quad R^{2}=0.928 .
\end{gathered}
$$

本文所研究的化合物种类多, $K_{\mathrm{Ow}}$ 值跨度为五个数量级以上, 而生物试验本身亦有较大的 不确定性, 因此 (9) 式和 (10) 式的相关性已相当显著,表 1 表明应用它们计算得出的毒性数据 与实测值吻合较好. 式中 $K_{\mathrm{OW}}$ 为辛醇 / 水分配系数, 表示化合物在生物体和水相中的分配倾 向, 具有较高 $K_{\mathrm{OW}}$ 的有机物的亲脂性较强. 式中的 $E_{\text {номо }}$ 为最高占有轨道的能量即为分子第

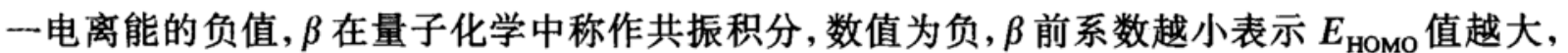
电子越易离去. 根据 (9) 和 (10) 式, 对于 $E_{\mathrm{HOMO}}$ 相近的化合物, $K_{\mathrm{OW}}$ 越大, 进人生物体的有机物 分子数目越多, 毒性越强, 例如多氯代苯的毒性顺序为: 五氯苯 $>$ 四氯苯 $>$ 三氯苯 $>$ 二氯苯

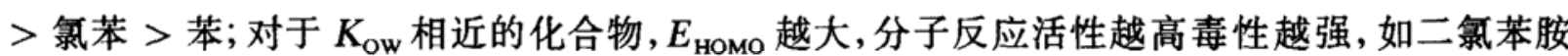
毒性 > 氯苯毒性, 皆与实测的毒性数据相符合. 由此可以看出, 方程式 (7)、(8) 成功地描述 了生物中毒机理; 同时还表明, 生物中毒分为两过程, 首先有机物分子透过细胞膜进人细胞 内, 其次有机物分子与细胞内分子发生作用。这两过程分别用有机物亲脂性参数 $\left(K_{\mathrm{ow}}\right)$ 及量 子化学参数 $\left(E_{\mathrm{HOMO}}\right)$ 来描述, 物理意义明确, 从而减少了 QSARs 法研究中参数选择的经验 性, 为进一步弄清生物中毒机理奠定了基础.

\section{参考文献}

[1] Blum, D. J. W., Speec, R. E., Environ. Sci. Technol., 1990, 24 (3): 284-293.

[2] 王连生,有机污染物化学,科学出版社, 北京, 1991, 3-71.

[3] Hoogen, G. V., Opperhuizen, A., Environ. Toxicol. Chem., 1988, 7: 213-219.

[4] Zhao Yuan-hui, Wang Lian-sheng et al., Chemosphere, 1993, 26 (11): 1971- 1979

[5] Devillers, J. et al., Chemosphere, 1987, 16 (6): 1149-1163.

[6] Stephenson, R. R., Watts, S. A., Environ. Publ., 1984, 36 (2): 95- 107.

[7] Verschueren, K., Handbook of Environmental Data on Organic Chemicals, Van N. R. Co., New York, 1983.

[ 8] Neely, W. B., Environ. Sci. Technol., 1979, 13 (12): 1506-1510. 